\title{
OF CARDIOVASCULAR PATHOLOGY
}

\author{
Centritto Enrico Maria ${ }^{1}$, Colombo Antonio ${ }^{2}$, Modugno Pietro
}

${ }^{I}$ MD, Vascular Surgery Specialist, Vascular Surgery Unit "John Paul II" Research and Care Foundation, "Catholic University of Sacred Heart" venue of Roma (Roma-IT).

${ }^{2}$ MSc, Cardiac Surgery Unit "John Paul II" Research and Care Foundation, "Catholic University of Sacred Heart" venue of Roma (Roma-IT).

${ }^{3}$ MD Vascular Surgery Rehabilitation Unit: John Paul II Research and Care Foundation "Catholic University of Sacred Heart" venue of Roma (Roma-IT); Operative Director UOSD of Vascular Surgery "Gemelli" Hospital Molise (Campobasso-IT).

KEYWORDS: Cardiovascular Rehabilitation, Physiotherapy, Rehabilitation, Anesthesiology

\section{ABSTRACT}

It is interesting to evaluate the role of cardiovascular and respiratory rehabilitation in patients undergoing carotid endo-arterectomy by observing the differences between patients undergoing general anesthesia and those under local anesthesia, with the aim of decreasing the risk of developing post-surgical complications, restore respiratory compliance and reduce user hospitalization times.

The aim of the study was to verify the effectiveness of rehabilitation treatment in a homogeneous group of 20 patients with symptomatic and non-symptomatic stenosis and / or $\geq 70 \%$ undergoing carotid endarterectomy, 10 under general anesthesia and 10 in loco-regional anesthesia, through a cycle of respiratory rehabilitation according to the rehabilitation protocol of the user undergoing cardiovascular surgery (Evidence Based Physiotherapy and Evidence Based Speech Therapy).

From the analysis of these two patients' groups, conclusions were drawn regarding the validity of physiotherapy treatment in the post-operative recovery of the patient undergoing carotid endarterectomy. Although the literature apparently showed no statistically significant differences between the two types of anesthesia, in our small study we recorded a difference between the two types of respiratory anesthesia. In Loco-regional Anesthesia, we found a stability in the levels of peripheral oxygen saturation at discharge compared to pre-operative. In the group of patients undergoing surgery by general anesthesia, peripheral saturimetry values worsened between pre-operative and discharge. The loco-regional anesthesia would seem to be more advantageous from a respiratory point of view than the general anesthesia in the general recovery of the patient. Also from a clinical point of view, in the patients of the loco-regional anesthesia group there was a lower perception of dyspnea, the absence of exacerbation of the cough reflex, a decrease in the difficulty in expectorating and a lesser presence of secretions in the airway.

\section{INTRODUCTION}

Cardiorespiratory rehabilitation is a branch of rehabilitation that has only risen in the last 20 years although cardiovascular diseases are among the main causes of death worldwide and the prospective forecasts for mortality and morbidity rates are not optimistic, lacking adequate information and prevention. In recent decades it has acquired the dignity of a scientific discipline with solid evidence, formulating its own evaluation methods and making therapeutic programs adapted to the different individual needs of patients. But despite the great progress in the diagnostic and therapeutic field, unfortunately this approach is often underestimated since it is considered more of a "final treatment" to resort to when traditional drug therapy has exhausted every resource. This interpretation is extremely reductive of its true potential. Hence the need to associate and use rehabilitation treatment in a cardiovascular surgery such as carotid endarterectomy, which among the main symptoms has: dizziness, visual disturbances (sensation of a veil in front of the eyes, which can worsen up to blindness and which may be reversible or lead to loss of a part of the visual field), paresis of the face, difficulty of speech, difficulty of movement, loss of strength of a limb or half of the body, disturbance of sensitivity (tingling, pain of a limb or half of the body), paralysis of a limb or half of the body, balance disturbances, fainting with or without loss of consciousness or even sudden death (Bond et al., 2004).

After surgery, as a result of surgical approach, bilateral paralysis of the vocal cords or the formation of a massive hematoma at the site of the surgical wound could occur (it is rare) and this will lead to respiratory failure (Bond et al., 2005).

In bilateral carotid endarterectomy interventions, reductions in ventilatory function have also been described with the loss of the normal respiratory response from denervation of the carotid chemoreceptors, which lose the ability to respond to the hypoxic stimulus causing an increase in the partial pressure of carbon dioxide in the blood arterial in resting conditions and a moderate arterial desaturation that requires the use of additional percentages of oxygen (Antonelli Incalzi et al., 1997).

It should not be forgotten that the baroreceptors are located at the bifurcation level of the common carotids and in the aortic arch, which are stimulated by the stretching of the vessel wall and are pressure receptors of the vascular system, involved in maintaining a 
pressure homeostasis.

Reasons that make the carotid endarterectomy intervention the ideal candidate for a pre and post-operative rehabilitation approach in order to re-establish the patient's clinical and functional conditions and improve the respiratory compliance compromised by the pathology (Ware \& Gandek, 1998).

\begin{tabular}{|l|l|l|l|}
\hline \multicolumn{4}{|c|}{ Table 07.I. Peripheral saturation of oxygen } \\
operated under General Anesthesia.
\end{tabular}

Tab. 1

\section{METHODS AND MATERIALS Patients}

In the period between May 2019 and September 2019 20 patients were enrolled in a randomized manner (Optimal Design Software), with different degree of carotid obstruction (symptomatic and non-symptomatic stenosis and / or $\geq 70 \%$ ) treated by carotid endarterectomy of which 10 undergoing surgery by general anesthesia, whose average age is 72.5 and 10 patients undergoing surgery by loco-regional anesthesia, whose average age is 73.6.

All participants signed the informed consent for the experimental procedure, which complies with the latest revision of the Helsinki Declaration.

The selection criteria for each patient evaluated in the study did not concern the difference in gender, weight, or age.

The following variables were assessed for each patient: the morphology and the percentage of plaque stenosis (homo and contralateral), the presence of preoperative neurological symptoms, cognitive and collaborative ability in the absence of severe dementia, the presence of cardiovascular and respiratory comorbidities and motor.

All patients underwent supra-aortic trunks before Eco Color Doppler, which allowed a flow and morphological evaluation of the extent of stenosis and a characterization of the plaque.

All surgery procedures were performed by a vascular surgeon with extensive.

- Inclusion criteria: Symptomatic and asymptomatic stenoses $\geq 70 \%$ ) (NASCET method) at the carotid bifurcation and / or origin of the internal carotid artery diagnosed by echocolordoppler or MRI angiography or CT angiography or angiography.

- Exclusion criteria: No exclusion criteria were set.

- Study design: The study was designed as an open, comparative, prospective controlled clinical trial.

- Recruitment time: The patients were enrolled in the time necessary for the intervention and its post-operative recovery.

- Duration of the study: Patients will be observed for a period of 4 days, taking into account the data obtained on the first pre-operative day, the first post-operative day and discharge.

- Functional and clinical evaluation: An initial and final evaluation is administered for all enrolled patients, following various accredited test and recording the most important data:

- Age

- Gender

- Hypertension

- COPD (Chronic Obstructive Pulmonary Disease)

- Heart disease

- CKD (Chronic Renal Disease)

- Type of surgery adopted.

Timing of the study:

- PHASE 1 (enrollment, T0): The patients included in the study are patients who met the inclusion criteria and who gave free informed consent.

- PHASE 2 (initial assessment, T0): For all patients, the collection of clinical data required for participation in the study and clinical and instrumental evaluation were performed.

- PHASE 3 (treatment, T1): Respiratory rehabilitation treatment.

- PHASE 4 (final evaluation, T2): Clinical and instrumental evaluation were repeated for all patients.

Guided questionnaire for the patient's medical history and quality of life: We have searched the literature for an accredited tool to meet our needs for:

- have specific anamnestic data in the respiratory rehabilitation field

- test the quality of life (QoL) and its variations after treatment.

\section{- EVALUATION}

Patient have been evaluated through saturimetry, in order to detect the percentage of saturation of oxygen in the blood. Particularly, the saturimetry allows to detect the $\mathrm{SO}_{2} \%$ value, which measure the percentage of oxygenated hemoglobin in relation to the total hemoglobin capable of transporting oxygen in the blood. A normal range of $\mathrm{SO}_{2}$ is typically between $94 \%$ and $98 \%$.

\section{Respiratory rehabilitation protocol}

Patients underwent a respiratory rehabilitation treatment through a program consisting of the following exercises:

- $\quad$ ACBT (Active Cycle of Breathing Techniques): It consists of periods of controlled breathing (BC), chest expansion exercises (TEE) and forced expirations (FET). The procedure must be adapted to the type of user by the number of repetitions for each exercise and once learned, it can also be used independently by the user.

- $\quad$ TEE (Thoracic Expansion Exercise): They are deep breaths with accentuation of the non-forced inspiratory and expiratory phase. After a passive exhalation to FRC, the user is asked for a slow inhalation from the nose up to TLC, with tele inspiratory apnea of about 3 seconds, followed by a non-forced exhalation with parted lips. This implies that:

- the slow, low flow inspiratory act facilitates the expansion of those areas of the parenchyma that require more time for the air to reach them 
- or it starts from an unforced expiration to $\mathrm{FRC}$, in order not to empty the lungs completely during exercise

- tele inspiratory apnea causes the air to redistribute from the fullest to the least full alveoli; better if carried out with the glottis open to allow a possible reintegration of air from the outside

- $\quad$ or the exhalation with parted lips causes the user to spontaneously apply a slight positive expiratory pressure.

- $\quad$ FET (Forced Expiration Technique): It consists of 1 or 2 Huff, or forced but non-violent exhalation, performed by contracting the abdominal muscles and keeping both the mouth and the glottis open. It starts from medium or low lung volumes, if you want to mobilize the most distal secretions, or from high volumes, if you want to have a proximal effect: the huff is always combined with a series of B.C;

- BC (Breathing Control): It is a calm breath, made with standard volume, using the lower chest, relaxing shoulders, and the upper chest. The BC is important to prevent bronchial spasms and breathing channels closure, allowing relaxing pauses for the patient (Marrocco-Trischitta et al., 2013).

- E.L.T.G.O.L. (Total Slow Exhalation with Glottis Open in Lateral Decubitus): The user lies in lateral decubitus position with the lung region that he intends to unblock in contact with the support surface and performs slow expirations from FRC to RV, keeping mouth and glottis open. If the user is unable to keep the glottis open, a cardboard mouthpiece can be used as a facility. This has a dual function: to guarantee the opening of the glottis and to amplify respiratory noises.

- The exhalation must be slow to avoid the increase in airway resistance caused by premature closure of the same. The physiotherapist can help the user by placing himself on the back side of this and, using the hand and the caudal forearm, exerts, starting from the lower abdominal quadrants, a diagonal thrust on the viscera, while the cranial hand stabilizes the supralateral hemithorax. This maneuver can be performed for 10/15 minutes per side. The user can be trained to perform this technique independently.

- Cough assistance: After-surgery users of thoracic or abdominal surgery cough assistance is performed, which consists of maneuvers implemented to produce an effective cough in the presence of pain and deficit of the abdominal wall due to the surgical act.

- Cough assistance is carried out through the containment of the abdominal wall and surgical wounds, manually or with bands / braces, and accentuating the flexion of the hips, to increase intra-abdominal pressure, thus ensuring better efficacy in the expulsion phase. Once the maneuver is learned, the user will be trained in self-management.

\begin{tabular}{|l|l|l|l|}
\hline \multicolumn{4}{|c|}{$\begin{array}{l}\text { Table 2. Peripheral saturation of oxygen (SO } \\
\text { erated under } \text { in patient op- }\end{array}$} \\
\hline & Entry & 1 Day After & Discharge \\
\hline Mean & 94,79 & 92,73 & 94,01 \\
\hline St. Dev. & 2,30 & 1,58 & 1,47 \\
\hline
\end{tabular}

Tab.2

\section{RESULTS}

In the indicated period of time, 20 patients underwent carotid endarterectomy were evaluated, 10 of whom underwent general anesthesia and 10 under loco-regional anesthesia; they were therefore divided into two distinct groups whose average age is 72.5 in the former and 73.6 in the remaining group. The comorbidities observed in both cases were hypertension, dyslipidemia, diabetes mellitus, COPD, and acute renal failure. The intervention procedures were all completed without technical difficulties. Mortality was $0 \%$ in both groups. Neither a permanent nor transient neurological deficit has occurred. There was no case of revision of hemostasis.

Among the values considered in our study are those of PCO2 (partial pressure of carbon dioxide), PO2 (partial pressure of oxygen) and SO2\% (oxygen saturation)

Among the parameters examined, we have reported those that showed the most significant variations.

The quantitative analysis of the data collected was performed using Office Excel software (Microsoft $\AA$, Redmond, Washington, USA), through which it was possible to create a graphical representation of the average variation of the parameters examined.

We report in Table 1, that of peripheral oxygen saturation in the group of patients after surgical approach with general anesthesia. The average of these values is $96.11 \%$ at the entrance with a standard deviation of $1.026807 ; 92.61 \%$ on the 1 st post-operative day with a standard deviation of 3.91931116 and $94.56 \%$ at discharge with a standard deviation of 1.73794259 . These values allow us to affirm that there is a stability of peripheral saturation (Table 1).

In patients after surgical approach with local anesthesia as can be seen from Tables 2 on the other hand, there was a slight decrease in peripheral saturimetry levels on the first post-operative day and an increase in peripheral oximetry compared to the first post-operative day; The average of these values is $94.79 \%$

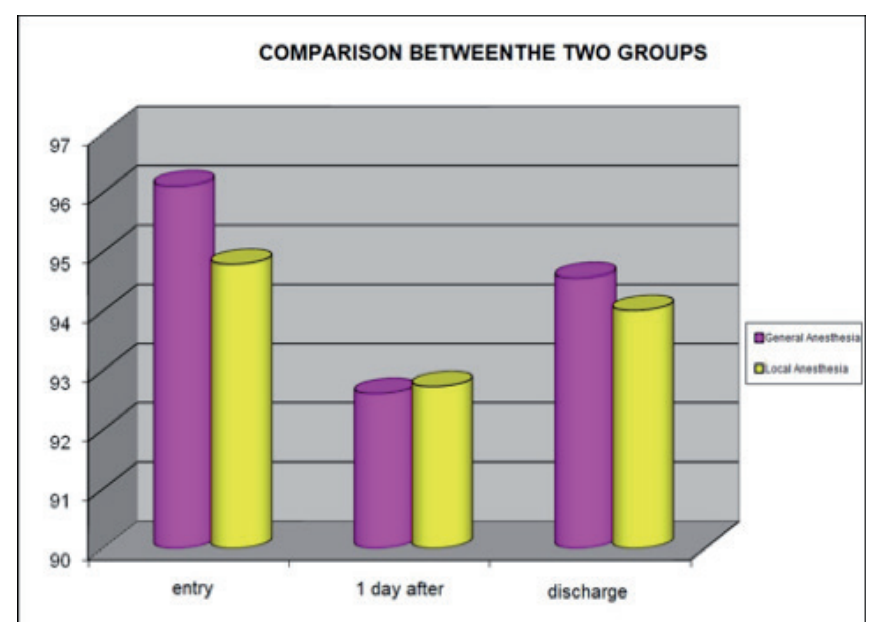

Fig. 1 - Graphic comparing the two groups of the study. 
at the entrance with a standard deviation of 2.30239; $92.73 \%$ on the 1 st post-operative day with a standard deviation of 1.588885 and $94.01 \%$ on discharge with a standard deviation of 1.471544918 (Table 2).

Therefore, as the comparison chart also highlights, loco-regional anesthesia would seem to be more advantageous from a respiratory point of view than general anesthesia (Figure 1).

\section{DISCUSSION}

This study conducted on 10 patients undergoing carotid endarterectomy under general anesthesia and 10 under loco-regional anesthesia, aimed to assess the feasibility and effectiveness of a treatment methodology applicable to the field of respiratory rehabilitation.

As there is no published data on the topic, it was decided to evaluate the effect of the technique on the main respiratory functional parameters and on some functional recovery parameters in twenty patients who underwent carotid thrombo-endo-arteriectomy (TEA)consecutively (Lloyd et al., 2004).

We searched for a potential effect by evaluating the values obtained from the blood gas analysis on the day of entry, on the 1st post-operative day and on discharge in the two groups of patients ( after surgical approach with general anesthesia and after surgical approach with local anesthesia) (Dardik et al., 2001). From the analysis of these 2 cases, in which we performed a prospective collection of data, we would like to be able to draw conclusions regarding the validity of physiotherapy treatment in the post-operative recovery of the patient undergoing carotid TEA.

Although the multicenter GALA trial and previous reviews of the Cochrane Library did not show statistically significant differences between the two types of anesthesia (Dellagrammaticas et al., 2008).

In our small study, we recorded a difference between the two types of respiratory anesthesia.

In Loco-regional Anesthesia, we found a stability in the levels of peripheral oxygen saturation at discharge compared to the pre-operative.

In the group of patients after surgical approach with general anesthesia, peripheral saturimetry values worsened between pre-operative and discharge.

Also from a clinical point of view, in the patients of the loco-regional anesthesia group there was a lower perception of dyspnea, the absence of exacerbation of the cough reflex, a decrease in the difficulty in expectorating and a lesser presence of secretions in the airways.

The Questionnaire as well as the rating scales, played a role of fundamental importance in framing and examining the subject's compliance with the continuation of respiratory exercises even at home.

\section{CONCLUSIONS}

This rehabilitation treatment, after a period of adequate training, provides an excellent degree of autonomy to the patient. Based on the scientific evidence, respiratory rehabilitation has a fundamental role in the multidisciplinary treatment of the patient undergoing cardiovascular intervention, in our study we have seen specifically in that of carotid endarterectomy. The rehabilitation intervention, in this case, is configured as a model of intensive rehabilitation and prevention of damage resulting from high-risk diseases.

The rehabilitation methodology has in fact proved to be feasible, reproducible, well accepted and tolerated by patients and seems to offer improvements from a respiratory point of view.

The data collected, although worthy of further studies and in-depth analysis through an increase in the number of cases, indicate that the patient undergoing carotid endarterectomy, especially after loco-regional anesthesia unlike patients undergoing general anesthesia, has the possibility full functional motor and respiratory recovery with effective rehabilitation treatment, aimed at improving alveolar ventilation, preventing and treating the obstruction of the airways by secretions.

\section{REFERENCES}

1. Antonelli Incalzi, R., Gemma, A., Landi, F., Pagano, F., Capparella, O., et al. (1997). Neuropsychologic effects of carotid endarterectomy. J Clin Exp Neuropsychol., 19(6), 785-94.

2. Bond, R., Rerkasem, K., Shearman, C. P., \& Rothwell, P. M. (2004). Time trends in the published risks of stroke and death due to endarterectomy for symptomatic carotid stenosis. Cerebrovascular Diseases, 18, 37-46.

3. Bond, R., Rerkasem, K., Cuffe, R., \& Rothwell, P. M. (2005). A systematic review of the associations between age and sex and the operative risks of carotid endarterectomy. Cerebrovascular Diseases, 20, 69-77.

4. Dardik, A., Minor, J., Watson, C., \& Hands, L. J. (2001). Improved quality of life among patients with symptomatic carotid artery disease undergoing carotid endarterectomy. J Vasc Surg., 33, 329-333.

5. Dellagrammaticas, D., Lewis, S. C., \& Gough, M. J. (GALA Trial Collaborators) (2008). Is heparin reversal with protamine after carotid endarterectomy dangerous? Eur J Vasc Endovasc Surg., 36(1), 41-44.

6. Lloyd, A. J., Hayes, P. D., London, N. J., Bell, P. R., \& Naylor, A. R. (2004). Does carotid endarterectomy lead to a decline in cognitive function or health related quality of life? J Clin Exp Neuropsychol., 26, 817-825.

7. Marrocco-Trischitta, M. M., Cremona, G., Lucini, D., Natali-Sora, M. G., Cursi, M., et al. (2013). Peripheral baroreflex and chemoreflex function after eversion carotid endarterectomy. J Vasc Surg., 58(1), 136-144.

8. Ware, J. E. Jr, \& Gandek, B. (1998). Overview of the SF-36 Health Survey and the International Quality of Life Assessment (IQOLA) Project. J Clin Epidemiol., 51(11), 903-912. 